\title{
图HAD
}

DOI: http://doi.org/10.22585/hospdomic.v3i3.80

\section{Implementación de una Aplicación móvil para trabajar con la Historia Clínica Electrónica de los pacientes en domicilio}

\author{
Implementation of a mobile application to work with \\ patient's Electronic Health Record at home
}

\begin{abstract}
Mercedes Díaz-Gegundez', Griselda Manzano Monfort² , Joan Garcia Vilches ${ }^{3}$, Isabel Gomez de Argila ${ }^{4}$

1. Atención Domiciliaria Integral, Corporació de Salut del Maresme i la Selva, Calella, Barcelona, España.

2. Hospitalización a domicilio, Corporació de Salut del Maresme i la Selva, Calella, Barcelona, España.

3. Tecnologías de la información, Corporació de Salut del Maresme i la Selva, Calella, Barcelona, España.

4. Hospitalización a domicilio, Corporació de Salut del Maresme i la Selva, Calella, Barcelona, España.
\end{abstract}

Correspondencia/Correspondence

Mercedes Díaz Gegundez

mdiaz@salutms.cat

Recibido/Received

27.06.2019

Aceptado/Accepted

06.07.2019

Conflicto de Intereses/Competing interest

La App ha sido desarrollada internamente por nuestra institución sin la participación de empresas o personal externo.

\begin{abstract}
Agradecimientos/Acknowledgments
A todos los profesionales de Hospitalización a Domicilio que han participado activamente en la implementación de la App

Al Dr. Guillem Paluzie por su ayuda metodológica

Financiación/Funding

El proyecto inicial de la App ganó un premio en las "Jornades de Recerca de la Academia de Ciencies Mediques" y con el dinero obtenido se compraron cuatro teléfonos móviles y se realizó un video del funcionamiento de la App.
\end{abstract}

CÓMO CITAR ESTE TRABAJO | HOW TO CITE THIS PAPER

Díaz-Gegundez M, Manzano Monfort G, Garcia Vilches J, Gomez de Argila I. Implementación de una Aplicación móvil para trabajar con la Historia Clínica electrónica de los pacientes en domicilio. Hosp Domic. 2019;3(3):203-15 


\section{RESUMEN}

Objetivo: Diseñar e implementar una aplicación móvil (App) que permitiera a los equipos de Atención domiciliaria el acceso seguro a la información médica relevante del paciente en el domicilio, registrar el seguimiento y realizar posteriormente la descarga automática de la información registrada a la Historia Clínica Electrónica, ahorrando tiempo a los profesionales y evitando errores de transcripción.

Método: Un grupo de trabajo multidisciplinar analizó las necesidades de los equipos de Atención Domiciliaria y conjuntamente con el Departamento de Tecnologías de la Información (TI) diseñó y desarrolló la primera versión de la App. Se realizó una prueba piloto que sirvió para solucionar incidencias y realizar algunas modificaciones y finalmente se implementó la App en Hospitalización a Domicilio (HAD). Después de un año de funcionamiento, se analizaron datos de utilización de la App, se calculó el tiempo estimado ahorrado en transcripciones y se realizó una encuesta de usabilidad.

Resultados: Durante el primer año de implementación de la App, el 86\% de los profesionales de HAD la utilizaron habitualmente y consideraron que suponía una mejora para su trabajo diario.

Las horas teóricas ahorradas en un año en transcripción de información fue de 256, que correspondería a 36,5 jornadas laborales.

Conclusiones: El uso de una App para consultar y registrar datos en la Historia Clínica Electrónica durante la visita en el domicilio ahorra tiempo a los profesionales y contribuye a evitar errores de transcripción.

Palabras clave: Aplicaciones Móviles; Registros Electrónicos de Salud; Servicios de Atención de Salud a Domicilio; Servicios de Atención a Domicilio Provisto por Hospital.

\section{ABSTRACT}

Objective: To design and implement a mobile application (App) that allowed Home Health Care Teams secure access to the patients relevant medical information, to record follow-up data at home and the automatic download of the data collected to the Electronic Health Record, saving professionals time and avoiding transcription errors.

Method: The Home Health Care Teams needs were analyzed by a multidisciplinary group and the first version of the App was designed and developed. Later, a pilot test was carried out to solve incidents and make some modifications. Finally, the App was implemented in Hospitalization at Home (HAD in Spanish). After one year of implementation, data about App use were analyzed, the saving in the transcriptions time was calculated and a usability survey was conducted.

Results: During the first year of implementation, $86 \%$ of the professionals used it on a regular basis and consider that it has been an improvement for their daily work.

The theoretical saved hours in a year in the medical information transcription were 256, which would correspond to 36.5 days (7-hour shifts).

Conclusions: Using an App to consult and record data in the patients Electronic Health Record at home avoids transcription errors and saves professionals' time.

Keywords: Mobile Applications; Electronic Health Records; Home Care Services; Home Care Services, Hospital-Based. 


\section{INTRODUCCIÓN}

El envejecimiento de la población y la mejora de la sanidad han comportado un incremento de la prevalencia de enfermedades crónicas y el número de pacientes pluripatológicos, lo que implica fragilidad, discapacidad y en ultimo termino, dependencia (1).

Para dar respuesta a las necesidades de esta población, se están desarrollando nuevas formas de atención, muchas de las cuales tienen como centro de operaciones el domicilio del paciente (2-4).

Durante las dos últimas décadas también se ha producido un considerable desarrollo tecnológico (bombas de infusión, monitores, aparatos de oxigenoterapia y nebulizadores portátiles) que permiten tratar en el domicilio procesos que anteriormente requerían de ingreso hospitalario.

Por otra parte, la evolución de las nuevas tecnologías y los dispositivos móviles están transformando la práctica clínica actual combinando funciones de comunicación y computación en un mismo aparato de dimensiones muy reducidas y muy fáciles de transportar. La masiva integración de dispositivos móviles se debe también al rápido desarrollo y calidad de las aplicaciones de software medico $(5,6)$.

Nuestra institución es un consorcio público que gestiona 3 de las 7 áreas básicas de salud del territorio, 2 hospitales, 3 centros sociosanitarios y 2 centros de rehabilitación, con una población de referencia de 195.000 habitantes.

Hace más de 15 años que se inició el desarrollo de una Historia Clínica electrónica (HCe) propia que actualmente está desplegada en todos nuestros centros, incluyendo las residencias geriátricas privadas que dependen de nuestras Áreas Básicas de Salud. Esto facilita enormemente la comunicación entre niveles asistenciales y mejora la atención de los usuarios.

De la misma manera, en 2005 se apostó por un modelo de Atención Domiciliaria transversal con equipos que se ocupan de las necesidades cambiantes de los pacientes con patología crónica y que contempla el domicilio como el lugar central de la atención. El Servicio de Atención Domiciliaria consta de diversos dispositivos asistenciales que atienden a grupos de pacientes según sus necesidades:

- Equipos de Atención Domiciliaria de Primaria: 7 enfermeras y 2 médicos de soporte que atienden a unos 700 pacientes con enfermedades crónicas que, por problemas de falta de movilidad, están incluidos en un Programa de Atención Domiciliaria de Atención Primaria.

- Equipos Domiciliarios de Curas Paliativas (PADES): 2 médicos, 4 enfermeras y 2 Trabajadoras sociales, que atienden unos 400 pacientes al año que precisan curas paliativas.

- Equipo de Soporte a Residencias Geriátricas: 1 geriatra y 2 enfermeras especializadas en geriatría, que dan soporte a los profesionales de las residencias geriátricas de la zona en la atención especializada de los pacientes institucionalizados. Son 12 centros con más de 900 plazas.

- Hospitalización a Domicilio (HAD): 3 médicos y 9 enfermeras hospitalarias que tratan a domicilio pacientes con patología aguda o crónica agudizada hasta su resolución y que generan más de 1.000 altas anuales; ver figura 1.

En total, nuestros equipos de Atención Domiciliaria, ofrecen atención sanitaria especializada a más de 2.500 pacientes en su domicilio cada año.

Aunque se habían realizado diversas pruebas para poder disponer de conexión on-line con la HCe del paciente desde el domicilio a través de ordenadores portátiles, no se consiguieron superar dificultades técnicas como la lentitud en la conexión por la cantidad de datos a cargar o la falta de cobertura 4G. Por lo tanto, estos equipos aún trabajaban con un resumen de la HC en papel que 
se desplazaba al domicilio. Una vez regresaban al centro sanitario se transcribían los registros a la $\mathrm{HCe}$.

Figura 1: Organigrama de Atención Domiciliaria

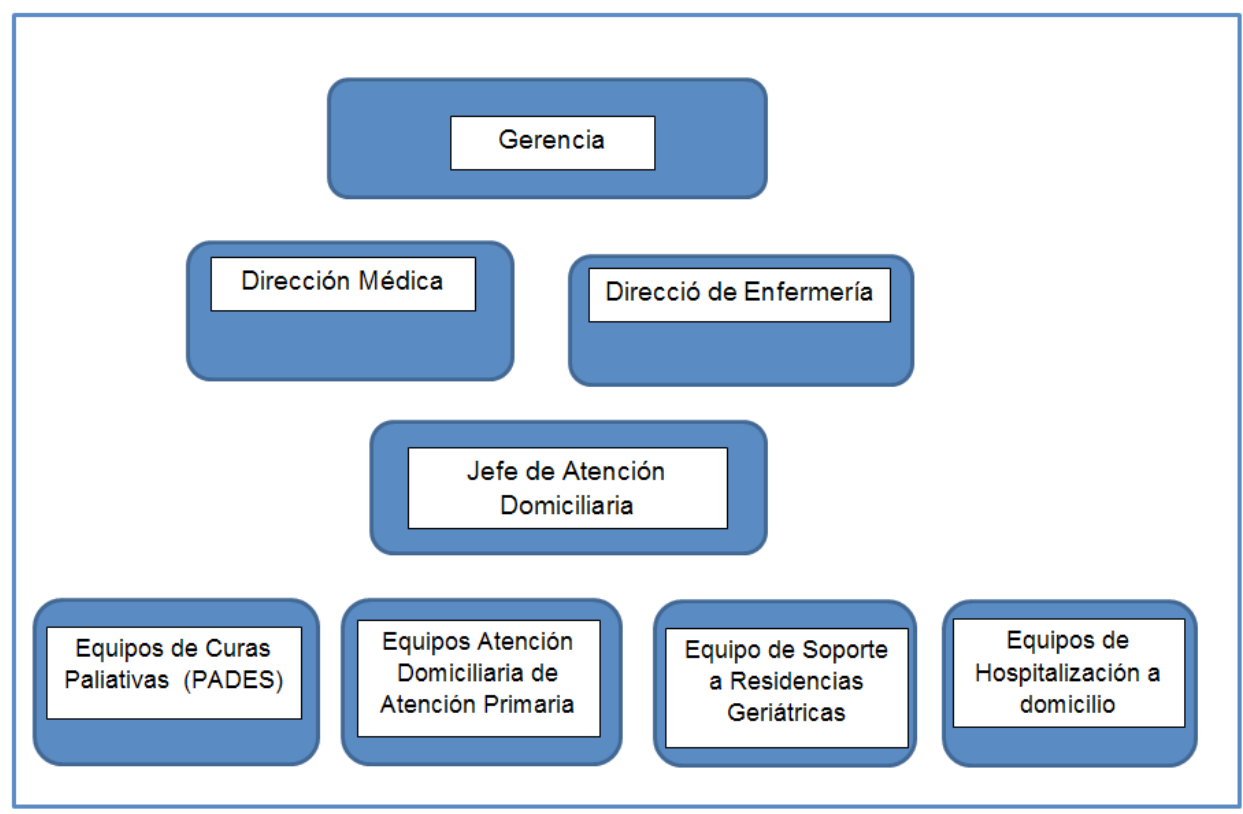

El principal objetivo del proyecto fue diseñar e implementar una aplicación móvil (App) que permitiera a los profesionales de Atención Domiciliaria disponer de accesibilidad segura a la información relevante del paciente, el registro del seguimiento en el domicilio y la posterior descarga automática de los datos recogidos a la $\mathrm{HCe}$, evitando errores y tiempo de transcripción.

\section{MÉTODOS}

\section{Análisis y diseño}

A finales de 2015, con la idea de desarrollar una App que diera respuesta a la necesitad de los equipos de Atención Domiciliaria de disponer de una plataforma móvil que les permitiera trabajar con la HCe del paciente desde el domicilio de manera ágil y segura, se creó un grupo de trabajo formado por el jefe de Tecnologías de la Información (TI), 1 técnico de informática, la jefe de Atención Domiciliaria, 1 enfermera y 1 médico del equipo de Hospitalización a Domicilio.

En una primera fase se realizó un análisis de las necesidades que se consideraban prioritarias:

- Poder disponer de la información básica de cada paciente en el domicilio para facilitar el seguimiento y ahorrar tiempo a los profesionales 
- Optimizar el tiempo y evitar errores dejando de hacer la transcripción de los datos, en papel en el domicilio y en la HCe una vez que se regresaba al centro sanitario.

- Agilizar el proceso de recogida de datos utilizando una plataforma digital adaptada

- La plataforma tenía que ser ágil y fácil de usar

- Se tenía que adaptar fácilmente a las características específicas de los diferentes equipos de domiciliaria

- Debía ser segura, interoperativa y exportable a otros servidores

En las siguientes reuniones y antes del diseño, se definieron las funcionalidades que debía tener la aplicación:

- Desde el Hospital, la App tenía que cargar y actualizar la lista de pacientes en el servidor, ya fuera desde la agenda de trabajo (en el caso de los Equipos de Domiciliaria de Atención Primaria o de Soporte a Residencias), desde el censo de pacientes ingresados (Hospitalización a Domicilio) o el censo de pacientes en programa (en el caso de los Equipos PADES)

- Una vez en el domicilio, desde la App, se tenía que visualizar la información relevante del paciente y permitir introducir datos, tanto en formato texto como estructurados

- La App tenía que permitir trabajar off-line en el domicilio para no depender de la conexión a internet y agilizar el proceso de consulta y la introducción de datos

- Una vez que se dispusiera de conexión wifi al llegar al centro de trabajo, se tenía que poder traspasar los datos recogidos a la HCe con una funcionalidad de sincronización

- La App no debía permitir realizar nuevas actualizaciones de datos en el dispositivo móvil si no se habían descargado previamente los últimos datos registrados

- La App debía permitir obtener la ruta de acceso al domicilio del paciente a través de la conexión directa con la aplicación para móviles de "Google Maps"

- Se tenía que poder conectar con el paciente a través del número de teléfono que consta en los datos personales de la HCe.

- Los profesionales deberían tener acceso al área de trabajo de la App con su clave personal

- La App tenía que permitir cambiar de usuario sin salir de la HC del paciente, para que los diferentes profesionales pudieran introducir datos durante la visita multidisciplinaria

- Los datos debían estar encriptados para garantizar la seguridad en caso de pérdida o robo del dispositivo

- Se tenía que poder explotar datos sobre carga y descarga de información desde la App y del uso del aplicativo por parte de los profesionales autorizados

Dado que los distintos equipos de domiciliaria tienen algunas características específicas, se decidió empezar por diseñar funcionalidades que fueran comunes a todos ellos, dejando para una segunda fase el desarrollo de funcionalidades más concretas para cada equipo.

Una vez desarrollada la App, se valoró implementarla en primer lugar en los equipos de Hospitalización a Domicilio ya que disponían de un censo de pacientes diario reducido (10 pacientes por equipo) y unas estancias cortas ( 7 días de media) lo que facilitó el seguimiento de su utilización y la valoración de su utilidad.

El dispositivo escogido para el desarrollo de la App fue el teléfono móvil, ya que su uso está muy extendido, son de medidas reducidas y actualmente disponen de pantallas bastante grandes. Los teléfonos móviles son fáciles de transportar y los profesionales de domiciliaria ya los utilizaban de forma habitual para contactar con los pacientes y con otros profesionales. Estos dispositivos debían disponer de conexión $4 \mathrm{G}$ y servicio de datos. 
También se decidió diseñar una aplicación que se pudiese instalar en el dispositivo móvil en vez de una aplicación basada en una Web, que requiriera funciones de conectividad a internet o los mensajes de intervención enviados desde un servidor, para no tener que depender de la recepción, la velocidad de conexión o el funcionamiento de un servidor remoto.

Por otro lado, y con el propósito de identificar los ítems de la Historia Clínica estadísticamente más utilizados por los profesionales de Hospitalización a Domicilio, se llevó a cabo un estudio observacional, descriptivo y transversal.

La muestra del estudio fue el número de consultas y registros realizados por los profesionales a los pacientes ingresados en HAD durante el mes de febrero de 2016 (10 pacientes en muestreo sistemático, comenzando por la primera alta y siguiendo por orden de 3 en 3). Las variables del estudio fueron los ítems de consulta y registro de los que dispone la HCe y que son los siguientes:

$\begin{array}{ll}\text { Datos personales } & \text { Petitorio } \\ \text { Exploraciones } & \text { Antecedentes } \\ \text { Radiología } & \text { Alergias } \\ \text { Estudios } & \text { Valoraciones } \\ \text { Laboratorio } & \text { Orden Mèdica } \\ \text { Biopsia } & \text { SIRE-Prescripciones } \\ \text { Citología } & \text { Planes de atención } \\ \text { Constates } & \text { Inmunizaciones } \\ \text { Curso Clínico } & \text { Interconsultas } \\ \text { Informes } & \text { Consultas Externas }\end{array}$

La extracción de datos se realizó a través de la base de datos de nuestro sistema informático (HIS). Se clasificaron los ítems disponibles por porcentajes de mayor a menor; ver tabla 1.

Tabla 1: Ítems de la historia clínica electrónica ( $\mathrm{HCe}$ ) más frecuentemente consultados por los profesionales

\begin{tabular}{|l|l|l|}
\hline Ítem & $\mathbf{N}^{\circ}$ de consultas por paciente & $\mathbf{N}^{\circ}$ de consultas por estancia \\
\hline Curso Clínico & 33,6 veces $\times$ paciente & 4,8 veces $\times$ estancia \\
\hline Orden médica & 30,2 veces $\times$ paciente & 4,3 veces $\times$ estancia \\
\hline Datos personales & 12,8 veces $\times$ paciente & 1,8 veces $\times$ estancia \\
\hline Tabla de constantes & 11,2 veces $\times$ paciente & 1,6 veces $\times$ estancia \\
\hline Informes & 10,2 veces $\times$ paciente & 1,4 veces $\times$ estancia \\
\hline Valoraciones & 9,6 veces $\times$ paciente & 1,3 veces $\times$ estancia \\
\hline Laboratorio & 6,8 veces $\times$ paciente & 0,9 veces $\times$ estancia \\
\hline Petitorio & 5,6 veces $\times$ paciente & 0,8 veces $\times$ estancia \\
\hline Radiologia & 5,1 veces $\times$ paciente & 0,7 veces $\times$ estancia \\
\hline
\end{tabular}


Una vez analizados los datos, el grupo de trabajo decidió cuales serían las funcionalidades que se incorporarían en la primera fase del desarrollo de la App teniendo en cuenta las necesidades de los profesionales y la complejidad del desarrollo.

Las funcionalidades finalmente escogidas fueron las siguientes:

- Visualización del censo de pacientes

- Acceso a los datos personales del paciente, con la dirección conectada a "Google Maps" y acceso a llamada directa pulsando sobre el número de teléfono de los datos personales.

- Visualización de los 20 últimos registros de curso clínico y poder registrar los nuevos

- Visualización de los 10 últimos registros de variables de constantes vitales y poder registrar las nuevas

- Visualización el último plan de tratamiento actualizado

\section{Desarrollo}

El Departamento de TI realizó el desarrollo técnico de la App utilizando una tecnología híbrida basada en Apache Cordova, que permite fácilmente adaptar la aplicación a las dos plataformas más utilizadas (Android y IOS). Inicialmente la App se desarrolló para Android, ya que es el sistema operativo utilizado en los teléfonos de nuestra empresa.

Se diseñaron y desarrollaron las diferentes pantallas. Los profesionales del grupo de desarrollo aportaron ideas en cuanto a distribución de los botones o definición de los campos a mostrar; ver figura 2.

La seguridad era un tema que preocupa mucho al grupo desarrollador y, por tanto, se trató específicamente durante las reuniones. Los siguientes son los puntos principales relativos a seguridad, protección de datos y accesos de la App que se definieron:

- El Departamento de TI sería el encargado de instalar la App en los teléfonos autorizados.

No estaría disponible en ninguna de las plataformas de descarga de App's

- El acceso al teléfono estaría asegurada con una clave privada y el teléfono estaría encriptado, lo que significa que no se podría conectar a ningún ordenador para extraer datos

- Si se produjese una perdida accidental del teléfono, se podrían borrar todos los datos e inhabilitar el teléfono de forma remota

- Toda la información utilizada temporalmente estaría encriptada y se borraría en el momento en que ya no fuese útil

- Las conexiones con servidores remotos se harían siempre sobre conexiones Wifi seguras y dentro de las propias instalaciones del centro. Es decir, la carga de los datos para que la App funcionase off-line no se podría realizar desde fuera del centro

- El mantenimiento de los teléfonos y de las personas autorizadas para poder utilizarlos se haría desde el Departamento de TI.

- El usuario, una vez accediese al teléfono y a la App con las claves correspondientes, debería poner el usuario y la clave personal para poder gestionar los pacientes asignados

- La trazabilidad de uso también estaría garantizada, debiendo quedar registradas todas las acciones que se realicen en la App y quien las realiza.

- La App debería ser capaz de reportar errores en la carga y descarga de los datos desde los servidores 
Figura 2: Principales pantallas de la App

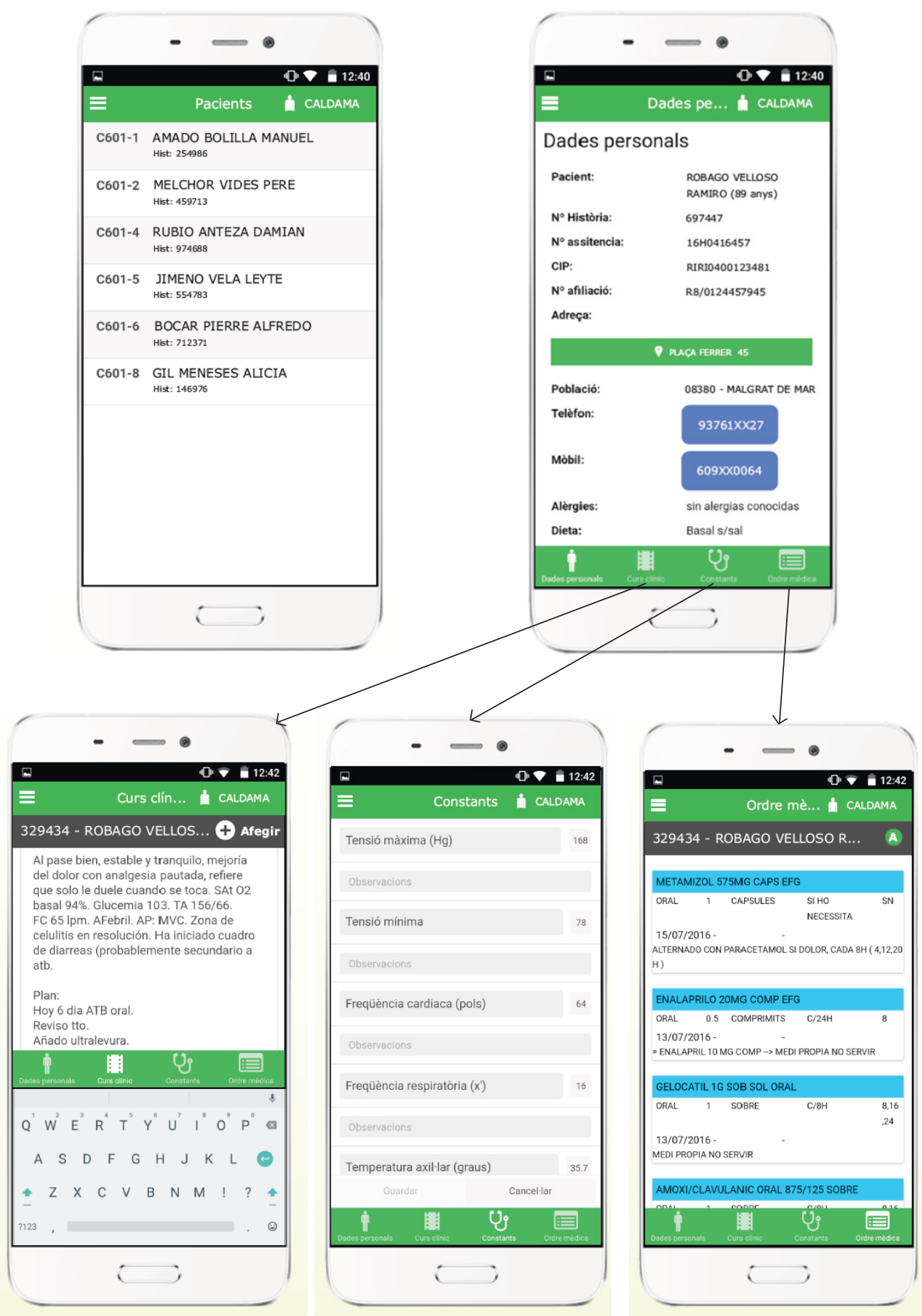




\section{Prueba piloto}

Una vez se dispuso de la primera versión de la App (mayo 2016), se realizó una prueba piloto con una muestra de conveniencia formada por profesionales de HAD (2 médicos y 3 enfermeras). A estos profesionales se les proporcionó un teléfono de 5,2 pulgadas con la App instalada, se les facilitaron los accesos y se les explicó el funcionamiento básico de la App. Se creó un "paciente ficticio" sobre el que pudieron probar todas las funcionalidades de la App.

Los participantes aportaron diversas ideas de mejora que se resumen a continuación:

- Cambiar el color de la cabecera (azul/verde) según profesional (médico o enfermera)

- Aumentar la medida de los caracteres de las constantes vitales

- Salto automático de casilla al rellenar los datos de constantes vitales, sin tener que pulsar "intro"

- Posibilidad de teclado horizontal

- Posibilidad de copiar un curso clínico anterior y modificarlo para ganar tiempo en el registro

- Que las constantes introducidas que estuviesen fuera de rango, apareciesen en rojo

- Posibilidad de que quedasen identificados diferentes tipos de curso clínico, en función de si se trataba de registrar una llamada telefónica, un visita de seguimiento o una gestión

- Posibilidad de registrar cursos clínicos con mensaje de voz

- Posibilidad de hacer fotos y que se traspasasen directamente a la $\mathrm{HCe}$

Todas las demandas se pudieron hacer efectivas excepto las tres últimas ya que requerían de una elevada inversión en tiempo de programación y supondría un retraso en la puesta en marcha del proyecto. Los profesionales que participaron en la prueba, valoraron que las funcionalidades ya desarrolladas podían suponer una mejora importante para su día a día.

En Junio de 2016 se realizó una prueba piloto con pacientes durante un periodo de un mes, los profesionales de HAD combinaron la recogida de datos convencional en papel con el uso de la App.

Se detectaron algunas incidencias de funcionamiento que fueron corregidas y solucionadas por el Departamento de TI.

\section{Implementación de la App en HAD}

En Septiembre de 2016 se dispone de la primera versión de la App ADIm (Atención Domiciliaria Integral móvil) finalizada y con garantías de uso y se implementa su utilización en los equipos de Hospitalización a Domicilio.

\section{RESULTADOS}

Después de un año de funcionamiento (de enero a diciembre de 2017) y según la explotación de los datos de uso de la App, el $86 \%$ de los profesionales de las unidades de HAD la utilizaron de manera habitual.

Al finalizar el primer año de implementación de la App, se realizó una encuesta a los profesionales de HAD (enfermeras y médicos habituales del servicio) para valorar aspectos de usabilidad. Se trataba de una encuesta creada por los desarrolladores basada en la selección de 8 preguntas extraídas de otras encuestas de valoración de aplicaciones (7-9). Son preguntas sencillas y formuladas para respuesta dicotómica y que se contestaron de manera anónima. 
Se enviaron 12 encuestas, a todos los profesionales autorizados con acceso a la App y se recibieron 11 encuestas contestadas; ver tabla 2.

Tabla 2: Encuesta de usabilidad

\begin{tabular}{|l|l|l|}
\hline Resultados encuesta usabilidad. ADIm. versión1 & SI & $\%$ \\
\hline ¿Consideras que las funcionalidades de la App se aprenden con rapidez? & 11 & $100 \%$ \\
\hline ¿Utilizas la App en tu rutina diaria de trabajo? & 11 & $100 \%$ \\
\hline ¿Consideras que la App es fácil de usar? & 11 & $100 \%$ \\
\hline ¿Es visualmente atractiva? & 8 & $73 \%$ \\
\hline ¿Se lee el contenido con facilidad? & 11 & $100 \%$ \\
\hline ¿Puedes introducir los datos rápidamente? & 11 & $100 \%$ \\
\hline ¿Te permite hacer alguna cosa que antes no podías hacer? & 9 & $82 \%$ \\
\hline ¿Crees que la App representa una mejora en tu trabajo habitual? & 11 & $100 \%$ \\
\hline
\end{tabular}

Los profesionales consideraron que la App era fácil de usar y útil en su rutina diaria. También creían que les había supuesto una mejora para su trabajo habitual.

El $30 \%$ de los encuestados opinaron que no era visualmente atractiva.

La aplicación tiene la capacidad de reportar errores que se puedan producir en la carga y descarga de datos para ser revisada por el Departamento de TI. Tampoco permite cargar nuevos datos si no se han descargado los anteriores para no perder información.

Durante 2017 solo se reportó un error en la descarga de datos debido a problemas en el servidor. Una vez solucionado, los datos se descargaron correctamente.

Entre Enero y Diciembre de 2017 se descargaron 5.201 cursos clínicos y 19.064 variables de constantes vitales, una media de 4 variables por paciente; ver figura 3.

Para calcular el tiempo teórico ahorrado en la transcripción, se midió el tiempo en minutos de la transcripción de 20 cursos clínicos de seguimiento y de las constantes vitales de 20 pacientes ingresados en un día concreto en las unidades de HAD por parte de 2 profesionales habituales del servicio (un médico y una enfermera).

De los datos obtenidos se extrapoló el tiempo necesario para transcribir un curso clínico y las constantes vitales haciendo la media del tiempo de las muestras que fue de 2,44 minutos para el curso clínico y de 0,55 minutos para cada grupo de constantes vitales (media de 4 variables por paciente). 


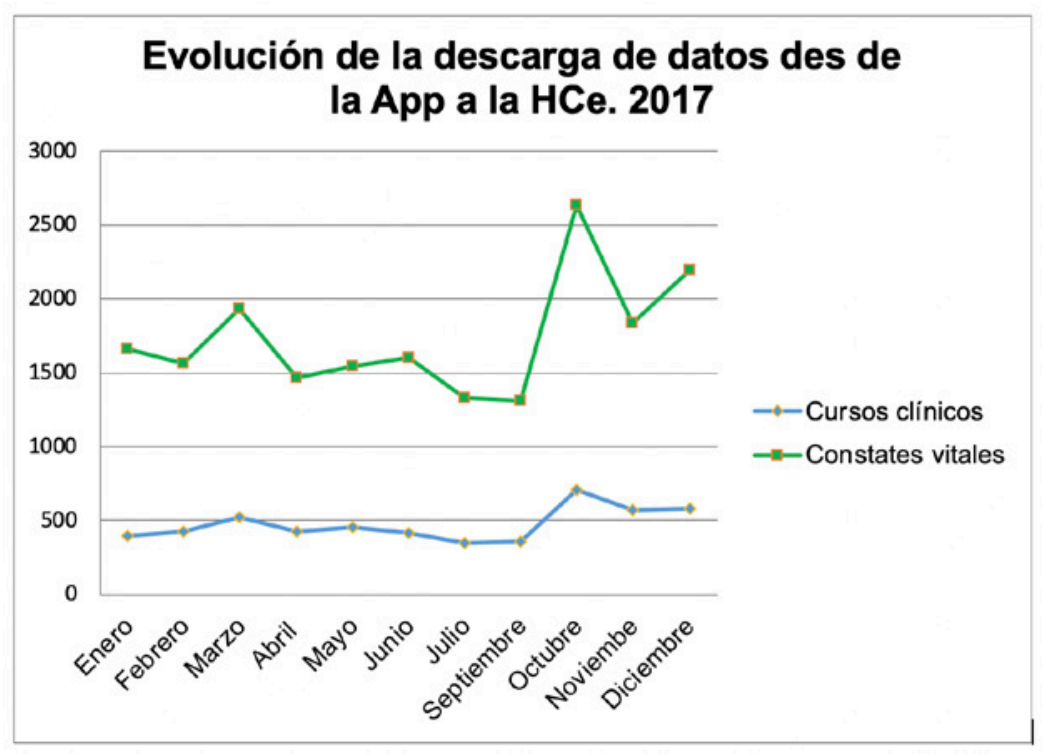

Teniendo en cuenta estos resultados se hizo un cálculo estimativo del tiempo ahorrado en 2017 en el proceso de transcripción de información por el uso de la App; ver figura 4.

Figura 4: Cálculo estimado del tiempo ahorrado en la transcripción de cursos clínicos y constantes vitales

\begin{tabular}{|l|}
\hline Cálculo del tiempo teórico ahorrado para transcribir cursos clínicos. Año 2017 \\
\hline 5.201 apuntes $\times 2,44^{\prime}$ de promedio para registrar $=12.690^{\prime} / 60^{\prime}=212 \mathrm{~h}$ \\
\hline Cálculo del tiempo teórico ahorrado para transcribir variables de constantes. Año 2017 \\
\hline 19.064 variables/4 variables por paciente $=4.776$ grupos de 4 variables \\
\hline 4.776 grupos de 4 variables $\times 0,55^{\prime}$ de promedio para registrar cada grupo $=2.622^{\prime} / 60$ ' $=44 \mathrm{~h}$ \\
\hline Total tiempo teórico ahorrado: 256 horas en un año \\
\hline
\end{tabular}

Las horas teóricas ahorradas en un año en la transcripción de la información recogida en el domicilio de los pacientes correspondería a 36,5 jornadas laborales de 7 horas. 


\section{DISCUSIÓN}

Consideramos que este trabajo ha demostrado que a través de una App, es posible la consulta y el registro de datos en la HCe desde el domicilio del paciente.

Al no tener que transcribir la información se evitan errores y se ahorra tiempo a los profesionales.

De igual manera, se aumenta la seguridad de los datos al evitar desplazar la HC en papel a los domicilios.

Los profesionales han integrado el uso de la App en su práctica diaria y consideran que supone una mejora para su trabajo habitual ya en esta primera fase de desarrollo.

Como limitaciones señalamos que no se puede disponer de toda la información que contiene la $\mathrm{HCe}$ del paciente en la App, lo que implica que, en ocasiones, los profesionales necesiten consultar algún dato que no pueden obtener hasta que regresan al centro, tal como ya pasaba antes de tener esta herramienta.

Actualmente las funcionalidades de registro de la App también son limitadas y los profesionales tienen que acceder a la $\mathrm{HCe}$ cuando regresan al centro para realizar peticiones de pruebas, cambios en el plan de tratamiento o rellenar valoraciones. Será necesario continuar el desarrollo de la App para incluir estas funciones que son de uso frecuente.

La App se ha probado en un grupo reducido de profesionales motivados, cuando se amplíe su uso al resto de equipos de domiciliaria se podrá comprobar si es aceptada tan positivamente. Pensamos que sí, dado que las demandas y necesidades de los equipos de domiciliaria son similares.

Las medidas de seguridad pueden resultar molestas para los usuarios, ya que hacen falta 3 claves diferentes para poder acceder a la información (la del teléfono, la de la App y la personal) pero creemos que estos pasos son completamente necesarios para mantener la seguridad de los datos.

En el futuro, se espera que la mejora de las conexiones inalámbricas permita también desarrollar la conexión on-line a la HCe a través de la App lo que mejoraría el acceso a la información contenida en la misma.

\section{BIBLIOGRAFÍA}

1. Departament de Salut. Pla de Salut de Catalunya 2011 - 2015. Barcelona: Generalitat de Catalunya; 2012.

2. Departament de Salut. Alternatives assistencials en el marc d'un sistema integrat: Models d'atenció domiciliaria i residencial als malalts crònics. Barcelona: Generalitat de Catalunya; 2012.

3. Departament de Salut. Criteris de planificació sobre hospitalització i alternatives assistencials en l'atenció a la cronicitat. Barcelona: Generalitat de Catalunya; 2014.

4. Díaz-Gegúndez M, Paluzie G, Sanz-Ballester C, Boada-Mejorana M, Terré-Ohme S, Ruiz-Poza D. Evaluación de un programa de intervención en residencias geriátricas para reducir la frecuentación hospitalaria. Rev Esp Geriatr Gerontol. 2011;46(5):261-4. DOI: 10.1016/j.regg.2011.03.001; PMID: 21944328

5. Ventola LC. Mobile devices and apps for healthcare professionals: uses and benefits. PT. 2014;39(5):356-64. PMID: 24883008 
6. Flodgren G, Rachas A, Farmer AJ, Inzitari M, Shepperd S. Interactive telemedicine: effects on professional practice and health care outcomes. Cochrane Database Syst Rev. 2015;(9):CD002098. DOI: 10.1002/14651858.CD002098.pub2; PMID: 26343551

7. Martínez-Pérez B, de la Torre-Díez I, Candelas-Plasencia S, López-Coronado M. Development and evaluation of tools for measuring the quality of experience (QoE) in mHealth applications. $J$ Med Syst. 2013;37(5):9976. DOI: 10.1007/s10916-013-9976-x; PMID: 24014255

8. Halsall V1, Rogers J, Witt J, Song S, Nguyen HDH, Kelly P. Development of a mobile App for family planning providers. MCN Am J Matern Child Nurs. 2017;42(5):263-8. DOI: 10.1097/ NMC.0000000000000354; PMID: 28816805

9. Harrison R, Flood D, Duce D. Usability of mobile applications: literature review and rationale for a new usability model. J Interact Sci [revista en Internet]. 2013 [accessed July 2, 2019];1(1):1. Available from: https://link.springer.com/article/10.1186/2194-0827-1-1 